\title{
Oral Health Care Education Regarding the Gingival Health, Knowledge, Attitude, and Behavior of the Pregnant Women
}

\author{
Nahid Ramazani ${ }^{1}$; Mohammad Aoyub Rigi Ladez ${ }^{2, *}$; Iraj Zareban ${ }^{3}$; Elmira Bagheri $^{4}$ \\ ${ }^{1}$ Department of Pediatric Dentistry, School of Dentistry, Children and Adolescent Health Research Center, Dental Research Center, Zahedan University of Medical Sciences, Zahedan, \\ IR Iran
${ }^{2}$ Department of Periodontics, Dental Research Center, School of Dentistry, Zahedan University of Medical Sciences, Zahedan, IR Iran \\ ${ }_{3}^{3}$ Health Promotion Research Center, Zahedan University of Medical Sciences (ZUMS), Zahedan, IR Iran \\ ${ }^{4}$ Zahedan University of Medical Sciences, Zahedan, IR Iran \\ ${ }^{*}$ Corresponding author: Mohammad Aoyub Rigi Ladez, Department of Periodontics, Dental Research Center, School of Dentistry, Zahedan University of Medical Sciences, Zahedan, \\ IR Iran. Tel: +98-5412423218, E-mail: dr.rigi@gmail.com
}

Received: April 13, 2014; Revised: August 13, 2014; Accepted: August 16, 2014

\begin{abstract}
Background:During pregnancy, physiological changes occur in body, which resulting results in oral health problems such as caries, tooth mobility, and pregnancy gingivitis. Inflammatory mediators might seriously affect the fetus and placenta. In addition, such materials stimulate the synthesis of prostaglandins, which would lead to preterm birth.

Objectives: This study aimed to evaluate the effects of two methods of anticipatory guidance presentation on the knowledge, attitude, and behavior of pregnant women regarding oral health care during pregnancy and the impact on gingiva.

Patients and Methods: Ninety pregnant women attended a Health Center in Zahedan, southern Iran, were divided into 3 groups of direct intervention, indirect intervention, and control during a 2-month quasi-experimental study. After examining their gingiva using Modified Gingival Index (MGI), a self-reported questionnaire was completed. Then, anticipatory guidance was presented to direct intervention group face-to-face using PowerPoint and to indirect intervention group using pamphlet. Shortly after intervention, the knowledge and attitude sections of the same questionnaire were completed by the two intervention groups. Two months later all sections of the same questionnaire (knowledge, attitude, and behavior) were completed by all participants. MGI was registered again. The changes in mean of scores of variables in these groups were compared during this 2-month period. The data were analyzed with SPSS at the significant level of 0.05 using Mann-Whitney U, Kruskal-Wallis and 1-way analysis of variance with post hoc tests.

Results: Intervention groups showed significant differences with the control group regarding scores of knowledge, attitude, behavior and MGI $(\mathrm{P}<0.05)$. Comparing intervention groups, a significant difference was observed in changing the mean scores of knowledge and behavior $(\mathrm{P}=0.023$ and $\mathrm{P}=0.020$, respectively).

Conclusions: This study showed the superiority of direct method of presentation in changing pregnant women's knowledge and behavior about oral health care.
\end{abstract}

Keywords:Education; Attitude; Behavior; Gingiva; Knowledge; Pregnant women

\section{Background}

During pregnancy, physiological changes occur in body, which results in oral health problems such as caries, tooth mobility, and pregnancy gingivitis (1-3). However, oral health during pregnancy can affect the pregnancy outcome too $(3,4)$. Preterm birth is one potential complication of pregnancy $(4,5)$. Dental caries might be the result of desire for sugar, also the risk of periodontal diseases increases by higher concentration of hormones (4, $6,7)$. Inflammatory mediators might seriously affect the fetus and placenta. In addition, such materials stimulate the synthesis of prostaglandins, which would lead to preterm birth $(7,8)$.

Unawareness of dental care importance is one of the factors challenging dental services during pregnancy (5). Nevertheless, most expecting women seek health care, and this period is an ideal time to offer a variety of health interventions (2). Moreover, oral health education dur- ing pregnancy can decrease the vertical transmission of microorganisms to infant's mouth (2). Thus, good oral hygiene during pregnancy not only improves oral and general health of the mother, but also contributes to the oral and general health of the infant (6).

Lin et al. examined the results of a dental educational program offered to pregnant women (9). In their study, knowledge about oral and gingival health showed a considerable improvement over time. In the research conducted by Cardenas et al. knowledge of pregnant women was improved shortly and 4 weeks after delivering anticipatory guidance (10). The results reported by Bahri et al. indicated that educational programs can improve knowledge, attitude, and behavior of pregnant women (11). In their study, Capasso et al. showed that knowledge about dental problems during pregnancy is insufficient (12). According to Keirse et al. study, only $28 \%$ of the pregnant

Copyright (C) 2014, Health Promotion Research Center. This is an open-access article distributed under the terms of the Creative Commons Attribution-NonCommercial 4.0 International License (http://creativecommons.org/licenses/by-nc/4.0/) which permits copy and redistribute the material just in noncommercial usages, provided the original work is properly cited. 
women reported "very good" oral health (13). As stated by Wandera et al., George et al. and Boggess et al. it is essential to improve oral health during pregnancy (14-16).

\section{Objectives}

To the best of our knowledge, no interventional study with such content has been ever conducted on pregnant women. We hypothesized that direct and indirect methods of guidance presentation can provide similar effects on pregnant women. Therefore, the present study attempted to offer an anticipatory guidance to expecting mothers and evaluate the effect of two methods of delivering the guidance on the knowledge, attitude, and behavior of pregnant women about oral health care and determine its effect on the clinical gingival status.

\section{Patients and Methods}

This quasi-experimental interventional study was approved by the Research and Ethics Committee of Zahedan University of Medical Sciences (No. 90-2269). A total of 90 pregnant women attended a health center in Zahedan, a city in southern Iran, were recruited during fall 2011. The study protocol conforms to the ethical guidelines of the 1975 Declaration of Helsinki. The initial sample size was 30 participants in each of direct intervention, indirect intervention, and control groups (totally 90), based on the power of 0.9 and the type I error of 0.05 obtained by using a previous study (11). The inclusion criteria were as follows: being 18-30 years old, having their first pregnancy, proficient in Persian, literate, and having at least one additional visit (two months later). The exclusion criteria included having medical problems, passing previously oral health courses, and having modified gingival index (MGI) score of 0 or 4 . The purpose and nature of the survey explained to each participant. All of them met the prerequisites and signed the detailed informed consent form. Easy and convenience method was employed for sampling these 90 participants. The individuals were randomly assigned to direct, indirect, and control groups by the sequence of entering health center in the listed order.

Data collection was done through clinical examination and self-reporting questionnaire. Having prepared the preliminary version of the questionnaire, face and content validity were confirmed using a panel of experts. Having applied the corrective feedback, the instrument was quantitatively evaluated. After receiving expert comments, the content validity ratio and content validity index were calculated. Items with content validity ratio > 0.62 and content validity index $>0.79$ were approved. To determine reliability, the questionnaire was completed by 30 individuals whose features were the same as the statistical population. The value of Cronbach $\alpha$ coefficient was $76 \%$ for questions of knowledge, $75 \%$ for questions of attitude, and $78 \%$ for questions of behavior. In the edited version of the questionnaire, three demographic questions (age, educational degree, and gestational age) were placed in the first section. The second to fourth sections covered 19 questions regarding knowledge, attitude, and behavior. The range of scores of knowledge, attitude, and behavior was 0 to 6,0 to 10, and 0 to 10, respectively. Beside these factors, MGI was recorded. The questions were scored as illustrated in Table 1.

This study was conducted in two stages with a 2-month interval. At stage I, MGI was determined through Ramfjord Teeth in sitting position using head light. For each tooth, four gingival units were graded, and then the score of each individual was calculated (15). A distal tooth was considered for individuals who lacked any of these teeth. Next, a questionnaire was given to the participants. Having gathered the complete questionnaire, the prepared guidance was delivered to each member of the intervention groups. The content of this guidance was arranged according to reference books and approved by four periodontists. For the direct intervention group, this guidance was presented face-to-face using a 20-minute PowerPoint presentation, while it was done in written form using the pamphlet for the indirect intervention group. These issues were addressed in the pamphlet and PowerPoint: 1) The impact of pregnancy on maternal oral health including pregnancy gingivitis, pregnancy tumor, dental caries, and dental erosion, 2) The impact of oral health on pregnancy outcome, including low birth weight and preterm delivery, 3) The impact of any nutritional deficiency during pregnancy on infant dental health, 4) Necessary recommendations regarding daily oral hygiene practices using dental brush, dental floss, and antimicrobial mouthwashes, scheduled dental visits, if necessary, attending for dental treatments preferably during the second trimester, avoiding excessive eating of sweets and snacks between meals, avoiding brushing immediately after vomiting and instead, using fluoride mouth wash and sugar free gum.

The members of indirect group were given enough time to read the pamphlet at the health center. Shortly after the presentation, sections two and three of the same questionnaire (knowledge and attitude) were filled out by subjects of the intervention groups. At the end of this stage, hygiene kits were awarded to each individual for expressing gratitude and encouraging participation in the second stage. In addition, the pamphlets were gifted to the participants received indirect intervention.

At stage II, having determined MGI, the same questionnaire was completed by participants of the control and intervention groups. In this stage, a finger toothbrush was given to each participant as encouragement to maintain infant oral health. Throughout the survey, none of the questions were answered. After collecting the questionnaires, however, all the questions were sufficiently responded. Also, in accordance with ethical issue, the pamphlet was given to members of the control and direct intervention groups. After collecting the questionnaire, responses were coded, reporting the scores of variables with sum of the codes. 
Data analysis was done by SPSS (V 19, SPSS Inc. Chicago, IL, USA). The Kruskal-Wallis test was used for comparing the scores of variables as well as the mean change of variables between groups. The Dunn post hoc test was used for pairwise comparison between the groups. The MannWhitney U test was used to compare intervention groups immediately after intervention. Also, a comparison of the mean score of MGI and its mean changes in three groups were done through 1-way ANOVA, while the pairwise comparison was done through post hoc Tukey. In each group, Friedman and Wilcoxon tests were administered respectively for comparison of variables over time (beginning, immediately after intervention, and the end of the study). Furthermore, the paired t test was used to compare MGI scores at the beginning and the end of the study.

\section{Results}

Out of 90 pregnant women enrolled in the study, 76 participants with an average age of $23.64 \pm 3.22$ years completed the study. The average age for the direct intervention group (28 people), indirect intervention group (23 people), and the control group (25 people) were $24.6 \pm$
3.6, 23.6 \pm 3.2 , and $22.7 \pm 2.5$ years, respectively. The education degree of the participants were diploma (36 people, $47.4 \%$ ), associate degree ( 8 people, $10.5 \%$ ), and bachelor's degree (32 people, $42.1 \%)$. In terms of gestational age, 21.05\% (16 people) of the study subjects were in the first trimester of their pregnancy, 53.95\% (41 people) of them were in the second trimester, and $25.0 \%$ (19 people) were in the third trimester. Moreover, in terms of age $(\mathrm{P}=0.101$, ANOVA), education degree ( $\mathrm{P}=0.076$, Chi-square) and gestational trimesters $(\mathrm{P}=0.071$, chi-square), there were no significant differences between groups. The more details are presented in Table 2 .

At the beginning, there was no significant difference in the mean score of knowledge, attitude, behavior and MGI. Shortly after intervention, a statistically significant difference was seen in the mean score of knowledge in intervention groups $(\mathrm{P}=0.025)$. At the end, however, there was a significant difference in the mean score of knowledge, behavior, and MGI among three groups $(\mathrm{P}<0.05)$. A significant difference was observed in these variables comparing the control with each of the intervention groups $(\mathrm{P}<0.05)$. Regarding the mean change in scores

Table 1. Score and Score Range of Each Section of the Questionnaire

\begin{tabular}{|c|c|}
\hline Sections and Score Range of Questions & Response and Score \\
\hline Knowledge (0-12) & $\begin{array}{l}\text { correct (1), I do not know (0), } \\
\text { incorrect (0) }\end{array}$ \\
\hline $\begin{array}{l}\text { 1. Which of the following items will increase the risk of tooth decay? A) Fruits, B) Sweet and sticky } \\
\text { materials, C) Vegetables, D) I do not know }\end{array}$ & \\
\hline $\begin{array}{l}\text { 2. If needed during pregnancy, when is the best time for dental treatment? A) First trimester, B) } \\
\text { Second trimester, C) Third trimester, D) I do not know }\end{array}$ & \\
\hline $\begin{array}{l}\text { 3. What is the ideal number of dental visits for receiving oral health consultation during pregnancy? } \\
\text { A) Once, B) Twice, C) Three times, D) I do not know }\end{array}$ & \\
\hline $\begin{array}{l}\text { 4. What is the best way for maintaining good oral hygiene during pregnancy? A) Tooth brush and } \\
\text { tooth floss, B) Mouthwash, C) Chewing gum, D) I do not know }\end{array}$ & \\
\hline $\begin{array}{l}\text { 5. Recurrent vomiting in pregnancy can lead to which of the following situations? A) Tooth wear, B) } \\
\text { Tooth pain, C) Gum disease, D) I do not know }\end{array}$ & \\
\hline
\end{tabular}
Attitude (5-15)

I agree (2), no comment (1), I disagree $(0)$

7. I believe that good nutrition during pregnancy is essential for healthy teeth.

8. I believe that good oral hygiene during pregnancy decreases dental caries and periodontal disease.

9. I believe that risk of dental caries is high in pregnant women.

10. I believe that bearing toothache is better than performing dental procedures or using the analge- I disagree (2), no comment sics during pregnancy.

11. I believe the dental procedures are better postponed until after delivery.

Behavior (0-10) (1), I agree (0)

12. In order to reduce the rate of dental caries during pregnancy; do you use sugar free gum?

13. Do you go to the dentist during pregnancy to get dental consultation?

14. Do you use antibacterial mouthwash during pregnancy?

15. If you need dental treatment during pregnancy, do you postpone it until after delivery?

16. Do you brush your teeth immediately after vomiting?

17. Do you use fluoride to control your dental caries?

18. How many times have you brushed your teeth in the past 24 hours?

three times (2), two times

(1.5), one time (1), no use (0)

19. How many times have you flossed your teeth in the past 24 hours? 
from the beginning to the end, there was a significant difference in all variables $(\mathrm{P}<0.05)$ (Table 3$)$.

Furthermore, there was a statistically significant difference regarding the mean change in scores of attitude and MGI only by comparing the control with the two intervention groups, while regarding the mean change in scores of knowledge and behavior, a statistically significant difference was observed by pairwise, comparing all three groups $(\mathrm{P}<0.05)$. Table 4 presents the pairwise comparison of the groups.

For each intervention group, there was a statistically significant difference in the mean of knowledge and attitude scores over time, comparing the beginning, shortly after the intervention, and the end of the study $(\mathrm{P}<0.05)$ (Table 5). Comparing the results at the beginning time and the end in the intervention groups, there was a significant difference between the mean of knowledge, attitude, behavior, and MGI scores except for the mean of attitude scores achieved by members of the indirect intervention group ( $\mathrm{P}$ $=0.113$ ). However, no significant differences were observed between the mean scores of the examined variables in the control group from the beginning to the end (Table 5).

There was no significant relationship between degree of education, knowledge, attitude, and behavior in the groups at different times. Similarly, no significant relationship was observed between the three variables of knowledge, attitude, as well as behavior and age in each group and time. There was also no relationship between changes in knowledge, attitude, and behavior scores in the study groups $(\mathrm{P}>0.05)$.

\begin{tabular}{|c|c|c|c|}
\hline \multirow[t]{2}{*}{ Variable } & \multicolumn{3}{|c|}{ Groups } \\
\hline & Direct intervention & Indirect intervention & Control \\
\hline \multicolumn{4}{|l|}{ Education degree } \\
\hline Diploma & $16(57.1)$ & $6(26.1)$ & $14(56.0)$ \\
\hline Associate degree & $1(3.6)$ & $3(13.0)$ & $4(16.0)$ \\
\hline Bachelors & $11(39.3)$ & $14(60.9)$ & $7(28.0)$ \\
\hline \multicolumn{4}{|c|}{ Gestational trimester } \\
\hline First & $3(10.7)$ & $4(17.4)$ & $9(36.0)$ \\
\hline Second & $14(50.0)$ & $15(65.2)$ & $12(48.0)$ \\
\hline Third & $11(93.3)$ & $4(17.4)$ & $4(16.0)$ \\
\hline
\end{tabular}

\begin{tabular}{|c|c|c|c|c|}
\hline Variable & Beginning & Shortly After Intervention & End & Change in Score $^{\mathrm{C}}$ \\
\hline \multicolumn{5}{|c|}{ Knowledge } \\
\hline Direct & $3.31 \pm 0.49$ & $5.37 \pm 0.73$ & $4.69 \pm 0.70$ & $1.38 \pm 0.72$ \\
\hline Indirect & $3.31 \pm 0.75$ & $4.78 \pm 0.91$ & $4.30 \pm 0.78$ & $0.99 \pm 0.67$ \\
\hline Control & $3.66 \pm 0.70$ & & $3.6 \pm 0.67$ & $-0.06 \pm 0.66$ \\
\hline PValue & $0.767^{\mathrm{d}}$ & $0.025^{\mathrm{e}}$ & $<0.001^{\mathrm{d}}$ & $<0.001^{\mathrm{d}}$ \\
\hline \multicolumn{5}{|l|}{ Attitude } \\
\hline Direct & $7.92 \pm 0.85$ & $9.00 \pm 0.96$ & $9.30 \pm 2.80$ & $1.38 \pm 0.94$ \\
\hline Indirect & $8.11 \pm 0.91$ & $9.30 \pm 0.53$ & $8.55 \pm 0.65$ & $0.44 \pm 0.84$ \\
\hline Control & $8.26 \pm 0.85$ & & $8.13 \pm 0.79$ & $-0.13 \pm 0.52$ \\
\hline PValue & $0.523^{d}$ & $0.712^{\mathrm{e}}$ & $0.233^{\mathrm{d}}$ & $0.016^{\mathrm{d}}$ \\
\hline \multicolumn{5}{|l|}{ Behavior } \\
\hline Direct & $4.36 \pm 2.21$ & & $7.16 \pm 2.02$ & $2.80 \pm 1.71$ \\
\hline Indirect & $5.13 \pm 4.75$ & & $6.85 \pm 1.58$ & $1.72 \pm 1.75$ \\
\hline Control & $4.16 \pm 1.89$ & & $4.42 \pm 1.92$ & $0.26 \pm 1.13$ \\
\hline PValue $^{\mathrm{d}}$ & 0.884 & & 0.001 & $<0.001$ \\
\hline \multicolumn{5}{|l|}{ MGI } \\
\hline Direct & $1.10 \pm 0.35$ & & $0.42 \pm 0.32$ & $-0.68 \pm 0.34$ \\
\hline Indirect & $1.11 \pm 0.37$ & & $0.44 \pm 0.36$ & $-0.67 \pm 0.23$ \\
\hline Control & $1.04 \pm 0.36$ & & $1.12 \pm 0.36$ & $0.08 \pm 0.26$ \\
\hline PValue $\mathrm{f}^{\mathrm{f}}$ & 0.717 & & 0.0004 & 0.0002 \\
\hline \multicolumn{5}{|c|}{$\begin{array}{l}\text { a Data are presented as Mean } \pm \text { SD. } \\
\mathrm{b} \mathrm{P}<0.05 \text {. } \\
\mathrm{c} \text { Difference of the scores of the end and the beginning. } \\
\mathrm{d} \text { Kruskal-Wallis test was used for comparing the mean scores of knowledge, attitude, and behavior and the mean change of scores between groups. } \\
\text { e Mann-Whitney U test was used for comparison between the two intervention groups for the mean scores of the knowledge and attitude shortly after } \\
\text { the intervention. }\end{array}$} \\
\hline
\end{tabular}




\begin{tabular}{|c|c|}
\hline Variable & PValue $^{\mathrm{b}}$ \\
\hline \multicolumn{2}{|c|}{ Change in the mean score of knowledge } \\
\hline Direct/control & $<0.001$ \\
\hline Indirect/control & 0.002 \\
\hline Direct/indirect & 0.023 \\
\hline \multicolumn{2}{|c|}{ Change in the mean score of attitude } \\
\hline Direct/control & 0.007 \\
\hline Indirect/control & 0.03 \\
\hline Direct/indirect & 0.534 \\
\hline \multicolumn{2}{|c|}{ Change in the mean score of behavior } \\
\hline Direct/control & $<0.001$ \\
\hline Indirect/control & $<0.001$ \\
\hline Direct/indirect & 0.020 \\
\hline \multicolumn{2}{|c|}{ Change in the mean score of MGI } \\
\hline Direct/control & $<0.001^{\mathrm{C}}$ \\
\hline Indirect/control & $<0.001^{\mathrm{c}}$ \\
\hline Direct/indirect & $0.856^{c}$ \\
\hline \multicolumn{2}{|c|}{$\begin{array}{l}\mathrm{a} P<0.05 \text {. } \\
\mathrm{b} \text { Dunn post hoc test was used for pairwise comparison between the } \\
\text { groups for the mean scores of knowledge, attitude, and behavior and } \\
\text { the mean change of these variables. } \\
\mathrm{c} \text { Tukey post hoc was used for pairwise comparison of groups for the } \\
\text { mean score of MGI and the mean change in score of this variable. }\end{array}$} \\
\hline
\end{tabular}

Table 5. Over Time Comparison of the Knowledge, Attitude (Beginning, Shortly After the Intervention, and the End), Behavior and MGI Scores (At the Beginning and the End) in the Study Groups a

\begin{tabular}{|c|c|}
\hline Group & PValue $^{b}$ \\
\hline \multicolumn{2}{|l|}{ Direct } \\
\hline Knowledge & $<0.001$ \\
\hline Attitude & $<0.001$ \\
\hline Behavior & $<0.001^{\mathrm{C}}$ \\
\hline MGI & $0.0004^{d}$ \\
\hline \multicolumn{2}{|l|}{ Indirect } \\
\hline Knowledge & $<0.001$ \\
\hline Attitude & $<0.001$ \\
\hline Behavior & $<0.001^{\mathrm{C}}$ \\
\hline MGI & $0.001^{d}$ \\
\hline \multicolumn{2}{|l|}{ Control } \\
\hline Knowledge & $0.693^{c}$ \\
\hline Attitude & $0.559^{c}$ \\
\hline Behavior & $0.205^{c}$ \\
\hline MGI & $0.107^{\mathrm{d}}$ \\
\hline \multicolumn{2}{|c|}{$\begin{array}{l}\mathrm{a} P<0.05 \text {. } \\
\mathrm{b} \text { Friedman test was used for comparing the variables of knowledge } \\
\text { and attitude over time in intervention groups. } \\
{ }^{c} \text { Wilcoxon test was used for comparing the variable of behavior in all } \\
\text { groups and the variables of knowledge and attitude in control group } \\
\text { from beginning to the end of the study. } \\
d \text { Paired t test was used to compare MGI scores at the beginning and } \\
\text { the end of the study in all groups. }\end{array}$} \\
\hline
\end{tabular}

\section{Discussion}

Comparison of the groups studied in the present research showed statistically significant differences in the mean scores of knowledge, attitude, behavior, and MGI. Regarding the mean change in knowledge and behavior scores, there was a statistically significant difference between the intervention groups. In the present study, MGI was considered as an objective index for investigating the effect of each educational method. In fact, such an index is quick and easy to use, and also convenient and tolerable for individuals (9). Lin et al. in their study, observed some improvement in the gingival index, which is consistent with the results of our study (9). With regard to the considerable changes in MGI scores, which can be used more reliably than the self-reporting behavior, it can be stated that the educational program was greatly influential in the intervention groups. In the present research, a comparison of the results at the beginning and at the end showed a significant difference between the mean scores of knowledge, attitude, behavior, and MGI in the direct intervention group, which implied that the educational program was positively influential when managed directly. Cardenas et al. in their study offering anticipatory guidance through PowerPoint display, found out that the knowledge of the examined population about oral health advanced within four weeks, which is consistent with the results of our study (10). Additionally, there was a significant difference between the mean scores of knowledge, behavior, and MGI in the indirect intervention group, while no obvious change was observed in attitude scores. Such discrepancy might signal the need for longer time spans in order to attain a dramatic change in attitude compared to indirectly-offered educational program. At the same time in this group, short-term behavior and MGI can be improved following knowledge progress. In Bahri et al.' study, there was a significant difference between knowledge, attitude, and behavior scores at the beginning and the end points (11). In our study, however, no significant difference was observed in the variables over time in the control group, which was expected.

As for the variable of knowledge and the subsequent changes, there was a significant difference between the intervention groups shortly after intervention. It seems that direct interaction, eye contact, body language and non-verbal communication between the trainer and the audience in the direct method of intervention and appealingly unique characteristics of PowerPoint such as its interesting and colorful presentation have led to a significant difference in the mean score of knowledge achieved by the intervention groups shortly after the intervention. This study indicated that the 2-month period eliminated the effect of such relationship and led to no significant difference in the mean score of knowledge at the end. There was, however, a significant difference in the mean score of knowledge (difference in the mean score of knowledge at the beginning and the end) among three groups. In this respect, the two intervention 
groups were significantly different from one another and the control, which particularly showed the positive influence of direct intervention on changing the knowledge score. In line with the findings of the present research, Bahri et al. found a significant difference between scores of knowledge achieved by the control and intervention group at the end of their study (11).

As for the variable of attitude, no significant difference was observed between the intervention groups shortly after intervention and among the three groups at the end of the study. On the contrary, Bahri et al. reported a significant difference between scores of attitude achieved by the control and intervention groups at the end of the study (11). Even though the time span of our study is similar to that of Bahri at al., the discrepancy might have originated from several contributing factors, including sample size, type of intervention, and the education method. Moreover, regarding the mean change in the score of attitude, there was only a significant difference between the control, and the two intervention groups. As for the mean change in scores of knowledge, the direct method is preferred over the indirect method. In fact, the mean change in the score of attitude in the short period of this study was not affected by the method of intervention; in other words, only longer time spans can properly show the effect of the education method on changed attitude scores.

Although the highest behavior score at the end belonged to the direct intervention group (with the indirect intervention and the control groups ranking lower, respectively), there was no statistically significant difference between the intervention groups, and there was a significant difference between the control group and two intervention groups. Bahri et al. in their study, observed a significant difference in the behavior scores achieved by the control and intervention groups (11). With regard to the pairwise comparison of the mean score change of the behavior, there was a significant difference between the groups. As mentioned earlier, the two intervention groups were significantly different from one another in the mean change of knowledge scores.

The MGI score achieved by the intervention groups at the end of the study was lower than that of the control and showed a significant difference. Moreover, there was a significant difference in the mean change in MGI score of the intervention groups and that of the control. In that respect, the two intervention groups had no significant difference from one another. Accordingly, it can be stated that educational program, regardless of the method, was effective on the studied population, which can ultimately improve the clinical gingival status with no preference between the two methods in terms of effectiveness.

To explain the significant difference in the mean change of behavior scores of intervention groups, despite the existence of such a difference in the mean change of MGI scores, it should be noted that not all items of the questionnaire were related to gingival health and a few of them covered dental health related behavior. Thus, the comparison of the two groups in terms of the mean change in behavior and gingival clinical status did not yield the same result.

Lin et al. reported that information presented verbally was more helpful in comparison with that in written form (9). Direct intervention in the present study, however, yielded higher scores, even though it had no significant difference from the indirect intervention regarding the mean change in attitude and MGI scores. In this research, there was no significant relationship in each group in terms of the mean change in knowledge, attitude, and behavior scores, which implied that progress in knowledge did not necessarily lead to better attitude and also desirable behavior. As explained earlier, a period lasting over two months might be required in order to create a correlation between the mean change of knowledge and attitude scores. Regarding the fact that there is no correlation between knowledge and behavior, it can be argued that participants disregarded all the information they had already gained. Although this study indicates, to some extent, that short-term improved behavior was affected by the improved knowledge, another reason for the lack of correlation between the mean change in knowledge and behavior scores might relate to several "no comment" responses in the knowledge part, particularly at the beginning and end of the study. Such a lack of correlation aroused concerns regarding the possible bias in responses of the participants who inclined toward social desirability or a pleasant answer or even resorted to random selection. Such a possibility, however, seems unlikely considering other findings and the fact that participants in different groups answered the questions similarly.

In our study, there was no significant relationship between the education degree and variables of knowledge, attitude, and behavior; neither was there such a difference between age and the mentioned variables. On the other hand, in other studies $(4,16)$ such relationships have been reported. The results obtained from our study might have originated from the narrow distribution of age and education degree, which disallowed studying those with lower and higher levels of education and age.

In the present research, two education methods were employed by one person, and the materials taught were the same. Clinical examinations were also done by the same person. The other strength of this study was allocating a control group to which intervention groups could be compared. Although the sampling was done randomly and each eligible pregnant woman attending the health center, had equal chance to enter the study, the final results could not be generalized to the entire population of the pregnant women, because many of them might have chosen to visit other facilities, including private offices, which can probably influence individuals' willingness to accept the training. Furthermore, unknown traits such as socioeconomic status, family income, and previous oral 
problems may have confounded the results. The other restriction was that 14 individuals were not accessible at the end. Nevertheless, our study can be regarded as one of the few studies examining education methods to deliver the guidance in pregnancy. Considering the restrictions, conducting a study to evaluate the long-term progress of changes in variables is suggested. In such a study, the effects of other education methods of oral health care can be examined.

\section{Acknowledgements}

The authors would like to thank all the individuals who participated in the study.

\section{Authors' Contributions}

The overall implementation of this study, including design, data management, data analysis, and manuscript preparation were the results of joint efforts of co-authors of this paper. All authors have made extensive contribution into the review and finalization of this manuscript.

\section{Funding/Support}

Herein, we appreciate the Deputy of Research and Technology of Zahedan University of Medical Sciences, Zahedan, Iran for financial supports. This article was based on a thesis submitted to the graduate faculty, Faculty of Dentistry, Zahedan University of Medical Sciences, in partial fulfillment of the requirements for the DDS degree (No. 90-2415).

\section{References}

1. Ritter AV, Southerland JH. Pregnancy and oral health. J Esthet Restor Dent. 2007;19(6):373-4.

2. Kumar J, Samelson R. Oral health care during pregnancy rec- ommendations for oral health professionals. $N$ Y State Dent J. 2009;75(6):29-33.

3. Rieken SE, Terezhalmy GT. The pregnant and breast-feeding patient. Quintessence Int. 2006;37(6):455-68.

4. Thomas NJ, Middleton PF, Crowther CA. Oral and dental health care practices in pregnant women in Australia: a postnatal survey. BMC Pregnancy Childbirth. 2008;8:13.

5. Lee RS, Milgrom P, Huebner CE, Conrad DA. Dentists' perceptions of barriers to providing dental care to pregnant women. Womens Health Issues. 2010;20(5):359-65.

6. Villa A, Abati S, Strohmenger L, Cargnel M, Cetin I. Self-reported oral hygiene habits and periodontal symptoms among postpartum women. Arch Gynecol Obstet. 2011;284(1):245-9.

7. Haumschild MS, Holloway SC. The impact of maternal oral health during pregnancy on the mother and her baby. Clinical Feature. 2010:26-9.

8. Straka M. Pregnancy and periodontal tissues. Neuro Endocrinol Lett. 2011;32(1):34-8.

9. Lin DL, Harrison R, Aleksejuniene J. Can a prenatal dental public health program make a difference? J Can Dent Assoc. 2011;77:b32.

10. Cardenas LM, Ross DD. Effects of an oral health education program for pregnant women.J Tenn Dent Assoc. 2010;90(2):23-6.

11. Bahri N, Iliati HR, Bahri N, Sajjadi M, Boloochi T. Effects of oral and dental health education program on knowledge, attitude and short-time practice of pregnant women (Mashhad-Iran). $J$ Mash Dent Sch. 2012;36(1):1-12.

12. Capasso F, La Penna C, Carcione P, Vestri A, Polimeni A, Ottolenghi L. [Oral health and pregnancy: promotion of oral health during the pre-natal training in the Latina province]. Ann Ig. 2011;23(2):137-45.

13. Keirse MJ, Plutzer K. Women's attitudes to and perceptions of oral health and dental care during pregnancy. J Perinat Med. 2010;38(1):3-8.

14. Wandera MN, Engebretsen IM, Rwenyonyi CM, Tumwine J, Astrom AN, Promise-Ebf Study Group . Periodontal status, tooth loss and self-reported periodontal problems effects on oral impacts on daily performances, OIDP, in pregnant women in Uganda: a cross-sectional study. Health Qual Life Outcomes. 2009;7:89.

15. George A, Johnson M, Blinkhorn A, Ellis S, Bhole S, Ajwani S. Promoting oral health during pregnancy: current evidence and implications for Australian midwives. J Clin Nurs. 2010;19(2324):3324-33.

16. Boggess KA, Urlaub DM, Massey KE, Moos MK, Matheson MB Lorenz C. Oral hygiene practices and dental service utilization among pregnant women. J Am Dent Assoc. 2010;141(5):553-61. 